\title{
Las Tecnologías de la Información y Comunicación (TICs) Aplicadas a la Comprensión de Gráficos en Cinemática
}

\author{
Sergio L. Ribotta, Marcela I. Pesetti y Sonia N. Pereyra
}

Universidad Nacional de San Luis, Facultad de Ingeniería y Ciencias Económico-Sociales, Avenida 25 de Mayo N 384, (D5730EKQ) Villa Mercedes, San Luis-Argentina

(e-mail: sribotta@fices.unsl.edu.ar, mpesetti@fices.unsl.edu.ar, sonperey@fices.unsl.edu.ar)

\begin{abstract}
Resumen
El objetivo del presente estudio es conocer el impacto que tiene el uso de las Tecnologías de la Información y la Comunicación (TICs) en procesos de enseñanza y aprendizaje en carreras de Ingeniería. Se analiza la implementación de estrategias de enseñanza que incluyen recursos tecnológicos para facilitar aprendizajes comprensivos de los contenidos disciplinares de Física. Esta investigación aborda el estudio de una experiencia sobre la implementación de una propuesta didáctica que incluye el uso de las nuevas tecnologías para propiciar aprendizajes comprensivos en alumnos del curso Física I de la Facultad de Ingeniería y Ciencias EconómicoSociales (FICES) de la Universidad Nacional de San Luis (UNSL). Se propone analizar el uso de recursos tecnológicos como parte de determinadas estrategias de enseñanza en entornos presenciales y el impacto que provocan en la comprensión de gráficos en cinemática, especialmente cuando se representan estos fenómenos físicos integrando imágenes y experiencias en tiempo real, lo que se concluye finalmente.
\end{abstract}

Palabras claves: TIC, Física, enseñanza, Ingeniería, aprendizaje

\section{Information and Communication Technologies (ICTs) to Understand Kinematics Graphs}

\begin{abstract}
The objective of the current study is to determine the impact of the use of Information and Communication Technologies (ICTs) to teach and learn in Engineering. It is analyzed how teaching strategies that involve technological resources foster comprehensible learning of the contents of Physics. This research serves to explore an experience with students of "Physics I" of the Facultad de Ingeniería y Ciencias Económico-Sociales, Universidad Nacional de San Luis (UNSL) in an environment where new technologies have been applied to facilitate the learning process. As a conclusion, the study aims at analyzing the use of technological resources as part of certain teaching strategies in face to face environments and their impact in understanding kinematics graphs. These physical phenomena are represented integrating images and experiences in real time.
\end{abstract}

Keywords: physics, teaching, engineering, learning, ICT 


\section{INTRODUCCIÓN}

Los profesores universitarios son concientes de que la incorporación de las TICs, en la enseñanza no deviene necesariamente en el desarrollo de aprendizajes comprensivos por parte de los alumnos. Se considera que estas novedosas herramientas tienen un gran potencial, siempre y cuando sean integradas a una propuesta didáctica basada en la definición de que es aprender y cómo se aprende, y por tanto qué es enseñar y cómo se enseña el conocimiento disciplinar.

Con el fin de conocer el impacto que tienen el uso de las Tecnologías de la Información y las Comunicaciones (TICs) en los aprendizajes de ciertos contenidos disciplinares de Física en carreras de Ingeniería, se analizó y comparó la implementación de recursos tecnológicos, tales como DataStudio y VideoPoint, como parte de determinadas estrategias de enseñanza en entornos presenciales y la influencia que producen en la comprensión de gráficos en cinemática, cuando se representan estos fenómenos físicos integrando imágenes, animaciones, simulaciones y experiencias en tiempo real.

\section{Aspectos teóricos}

La presente propuesta didáctica que involucra el uso de las TICs, se fundamenta en las concepciones de aprendizaje significativo y comprensivo, conocimiento situado y distribuido, y de la mediación instrumental, como aportes provenientes del Constructivismo y de la Teoría Sociohistórica, cuyos principios permiten interpretar los procesos de enseñanza y aprendizaje y desarrollar las prácticas docentes.

Considerando la concepción del aprendizaje, las teorías constructivistas sostienen que las personas aprenden interactuando con los objetos y con otros sujetos en el marco de una cultura, en esa interacción los sujetos modifican la realidad (no literalmente sino atribuyéndole sentido) a la vez que se modifican los esquemas del sujeto, siendo los esquemas representaciones de una situación concreta o de un concepto que permite manejarlos internamente y enfrentarnos a situaciones iguales o parecidas en la realidad (Castorina, 1998).

En este proceso de interacción con el objeto de conocimiento Ausubel concibe al aprendizaje, como significativo, en tanto es un proceso de adquisición de significados a través del establecimiento de relaciones sustantivas entre el conocimiento previo y el nuevo material de aprendizaje. Es por ello, que considera a la exposición organizada de contenidos como un instrumento muy eficaz para conseguir una comprensión adecuada por parte de los alumnos. En este sentido, sugiere seleccionar y estructurar los contenidos de modo de favorecer la comprensión, teniendo en cuenta, no sólo el punto de vista de la disciplina, sino considerando el nivel de dificultad que le ofrece al alumno (Vélez, 2002).

Según Coll (1990), para Ausubel, aprender es sinónimo de comprender. Por ello, lo que se comprenda será lo que se aprenderá y recordará mejor porque quedará integrado a nuestra estructura de conocimientos El papel que cumple la enseñanza del docente es fundamental para generar este tipo de aprendizajes ya que, la intervención pedagógica ayuda a generar y desarrollar los procesos de aprendizaje.

La intervención del docente opera como mediación social, en términos de Vigotsky y las herramientas tecnológicas que utiliza en esa intervención, son mediaciones instrumentales que pueden facilitar los aprendizajes de los alumnos Vélez, 2002).

Vigotsky le atribuye gran importancia a la acción del ambiente social en el desarrollo del pensamiento hacia niveles superiores y al papel que cumplen los instrumentos psicológicos, mediadores. Considerando que la actividad, como acto instrumental, es el motor del desarrollo cultural, en tanto el hombre realiza un esfuerzo activo, actuando sobre la realidad para adaptarse a ella transformándola y transformándose a sí mismo a través los instrumentos mediadores. Esta mediación se lleva a cabo a través de herramientas (mediadores simples) y signos (mediadores más complejos) que actúan sobre el sujeto (Vélez, 2002). 
El uso de determinados instrumentos psicológicos transforma la capacidad de resolución de las tareas, amplificándola. Las diversas tecnologías representacionales modifican profundamente los esquemas de pensamiento al ser interiorizados.

Desde esta perspectiva, el aprendizaje es una construcción social, una experiencia compartida que vincula la acción y la representación. En este proceso el docente orienta al alumno, para que logre aprendizajes cada vez más avanzados y logre autonomía, según Vélez (2002) lo que Bruner, denomina andamiaje, que se refiere a la acción que puede desarrollar el adulto para llevar al joven de su nivel actual de conocimientos, a uno potencial, más elevado, transitando por la zona de desarrollo próximo generando un progresivo nivel de autonomía.

Considerando que el aprendizaje, como construcción social, se genera en interacción con otros y en cuyo proceso la orientación del docente acompaña la apropiación de conocimientos, Bruner plantea que el conocimiento es una acción situada y distribuida. No está ni en la cabeza ni en el exterior, está situado, en tanto que forma parte de un mundo cultural, sin asignarle un lugar específico, sino en las relaciones con múltiples objetos culturales y naturales que entabla el sujeto. Por ello, el conocimiento también se concibe como distribuido entre los diversos elementos y sujetos que participan en las interacciones, pero los objetos no como "lugares" del conocimiento, sino que los objetos se presentan mediados por las intenciones, por los saberes previos y por las acciones consecuentes. En este sentido, Salomón (2001) considera que la cognición humana posee propiedades distribuidas y resulta de procesos relacionales y sociales, se encuentra en el entrono, es una dinámica en red destacando el carácter interactivo de toda información y el carácter complejo, dinámico y abierto de la comunicación como espacio de construcción del conocimiento.

Desde esta perspectiva, según Vélez (2002) los factores tecnológicos, sociales y culturales no sólo sirven como recursos externos de estimulación, sino como herramientas que participan en el conocimiento, es decir como fuente y como vehículo del pensamiento. También las cogniciones se distribuyen simbólicamente: palabras, diagramas, gráficos, tablas o mapas conceptuales son medios de intercambio entre la gente (Perkins, 1993).

En este sentido, un programa interactivo, que tiene en cuenta el nivel de conocimientos previos, las capacidades de los estudiantes y genera procesos comprensivos se instaura como una modalidad adecuada para contribuir a la construcción del conocimiento.

El análisis que a continuación se realiza de la experiencia intenta demostrar como el uso de determinados recursos tecnológicos en una estrategia didáctica, contribuyen a mejorar la comprensión de los conceptos disciplinares. Específicamente, demuestra la influencia de las representaciones externas (imágenes, animaciones, simulaciones) utilizadas para registrar, analizar y explicar el fenómeno del movimiento, (Pontes, 2005) destinadas a mejorar la comprensión de conceptos de la cinemática a través de la interpretación de gráficos de cinemática.

\section{METODOLOGÍA}

Este estudio tiene como principal propósito, conocer el impacto que tiene el uso de las nuevas tecnologías en la comprensión de los gráficos de cinemática en los alumnos y alumnas de las carreras de Ingeniería en el curso de Física I de la FICES-UNSL. Considerando que la comprensión de algunos fenómenos físicos se dificulta con el análisis de figuras presentadas en material didáctico estático, el uso de las Tecnologías de la Información y la Comunicación (TICs) se presenta como una alternativa apropiada para enfrentar este desafío, en tanto permitiría construir escenarios reales y virtuales cognitivamente cercanos a la realidad física en distintos registros, como el simbólico, en cinemática.

Los objetivos específicos que se derivan de este propósito, se orientan a:

- Analizar el uso de determinados recursos tecnológicos como parte de una estrategia de enseñanza que intenta facilitar aprendizajes comprensivos.

- Determinar si el uso de representaciones externas (imágenes, animaciones, simulaciones, experiencias en tiempo real) ayudan a comprender conceptos de física (interpretación de gráficos en cinemática). 
La población de estudio se constituyó sobre la base de 86 alumnos que cursaban Física I durante el año lectivo 2006, lo que permitió conformar tres grupos de análisis. Con el fin de establecer grupos de trabajo homogéneos se valoraron diferentes aspectos de cada alumno, estableciéndose para ello las siguientes variables a considerar: valoración del rendimiento de los cursos previos a la asignatura Física I (Curso de Ingreso de Física y asignaturas del primer cuatrimestre, Algebra I y Análisis Matemático I), sexo de cada alumno (masculino y femenino), carrera a la que pertenecen (Ing. Química, Ing. en Alimentos, Ing. Electromecánica, Ing. Industrial e Ing. Electrónica) y un test para medir la actitud hacia la Física (Redish et al., 1997). Esta metodología permitió conformar en todos los casos que cada grupo estuviera integrado por la misma cantidad de alumnos y alumnas de todas las carreras de ingeniería involucradas, y a su vez que conservaran uniformidad los además aspectos entre los distintos grupos (rendimiento académico y actitud hacia la física).

En la fase inicial de la investigación participó un grupo de aproximadamente ochenta y seis (86) alumnos y alumnas que cursaron la asignatura Física I del primer año de las carreras de Ingeniería Electromecánica (11), Ingeniería Electrónica (13), Ingeniería Industrial (36), Ingeniería Química (15), Ingeniería en Alimentos (11) de la Facultad de Ingeniería y Ciencias EconómicoSociales (FICES) de la Universidad Nacional de San Luis (UNSL) durante el año 2006.

La cantidad de alumnos y alumnas que participaron regularmente de todas las actividades correspondiente a la experiencia, fueron 62 alumnos, distribuidos en cada grupo de la siguiente manera: Control: 21 alumnos, DataStudio: 20 alumnos y Videopoint: 21 alumnos

Para realizar la experiencia de análisis del uso de las TICs, se seleccionó el tema comprensión de gráficos de cinemática considerando dos factores fundamentales:

- Constituye un tema relevante dentro de la Física, siendo una aplicación que abarca íntegramente la mayoría de los conceptos relativos a la cinemática (sistema de referencia, trayectoria, movimiento, posición, desplazamiento, velocidad, aceleración, y fundamentalmente el carácter vectorial de las magnitudes -velocidad y aceleración-, representación gráfica, etc.). Si bien es frecuente su tratamiento en la asignatura (se lo aborda en cinemática, dinámica y energía), pero generalmente no se alcanza a profundizar conceptualmente sobre el mismo. Al estudiante le cuesta asociar que la velocidad y la aceleración son magnitudes vectoriales, analizar la velocidad como la razón de cambio de la posición y la aceleración como la razón de cambio en la velocidad (cambio vectorial), etc. (Beichner, 1994; McDermott et al., 1987; Arons, 1990).

- Existen diferentes software y hardware asociados (DataStudio, VideoPoint, Physlets, Interactive Physics, etc.) de la más amplia calidad con los cuales se pueden abordar fenómenos físicos con distintos niveles de profundidad. En el caso particular del presente trabajo solamente se hace referencia al análisis de dos de estas estrategias didácticas, DataStudio y VideoPoint. En experiencias posteriores, realizadas durante los años 2007 y 2008, se incorporó un grupo adicional que estudió el impacto que tiene la aplicación de los Physlets.

La experiencia se inicia luego del desarrollo de los aspectos teóricos del tema interpretación de gráficos de cinemática con un tratamiento matemático (analítico) dictado por el profesor responsable de la asignatura a todos los grupos simultáneamente. Luego, cada grupo individualmente (experimentales y de control) participó de una clase complementaria a la teoría desarrollada por otro profesor durante un periodo de aproximadamente 50 minutos.

Esta instancia estuvo constituida por diversas actividades: a) Introducción (breve revisión del desarrollo teórico), b) Visualización y explicación del fenómeno, c) Representación del mismo, con Videopoint, DataStudio (grupos experimentales) o pizarrón (grupo control) d) Visualización de los resultados obtenidos e) Análisis de resultados, f) Formulación de conclusiones y g) Análisis de otras aplicaciones similares.

Por una parte, los grupos experimentales, utilizaron recursos tecnológicos, tales como Videopoint y DataStudio. Se trató de crear un ambiente virtual, con la inclusión de gran cantidad de imágenes, como parte del trabajo del grupo. Por otra parte, con el grupo control el apoyo teórico 
se realizó, utilizando recursos de uso tradicional, fundamentalmente desde el trabajo con algunos esquemas y gráficas realizadas en el pizarrón.

Para ambos grupos de estudio (experimentales y de control) la evaluación de la instrucción se realizó mediante la aplicación de un diagnóstico de respuestas múltiples al inicio (pretest) y al final de la instrucción (postest). Este cuestionario constituido por 21 preguntas de respuestas múltiples corresponde al Test de Comprensión de Gráficos de Cinemática (TUG-K) (Beichner, 1994). Con los datos obtenidos a través de esta metodología permitió determinar la ganancia normalizada (Hake, 1998) que tiene la aplicación de un recurso didáctico, posibilitando además poder comparar el grado de logro de una estrategia educativa en distintas poblaciones, independientemente del estado inicial de conocimiento.

La experiencia consiste en mostrarle al alumno, independientemente del grupo, la misma situación referida a un mismo fenómeno, es decir todos observaron como se deslizaba un carrito en un sentido u otro sobre una riel (Figuras 1 a 5) o el movimiento de una pelota en el aire (Figura 6), lo que cambio entre el grupo control y los experimentales fue el recurso didáctico que se empleó para registrar, analizar y explicar el movimiento.

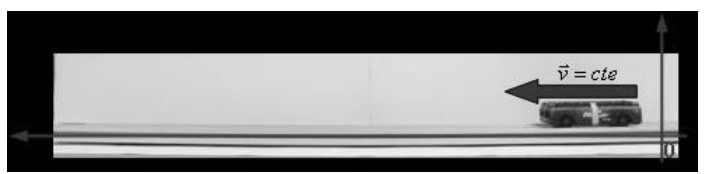

Fig. 1: Movimiento rectilíneo de un carrito con velocidad constante alejándose del origen del sistema de referencia (MRU).

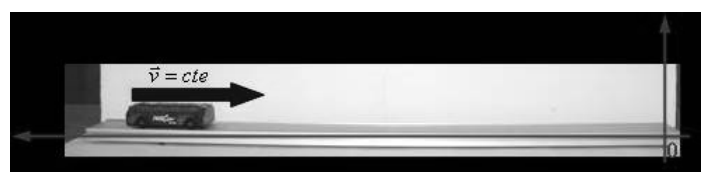

Fig. 2: Movimiento rectilíneo de un carrito con velocidad constante acercándose al origen del sistema de referencia (MRU).

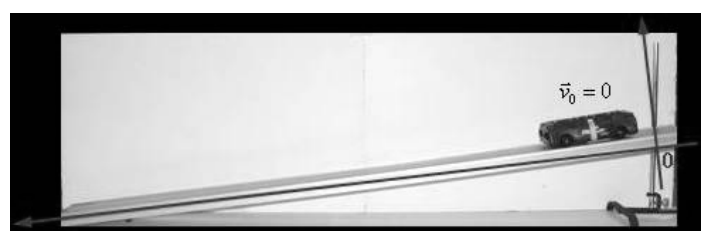

Fig. 3: Movimiento rectilíneo de un carrito con velocidad variable alejándose del origen del sistema de referencia (MRUA, análisis solamente del movimiento de bajada).

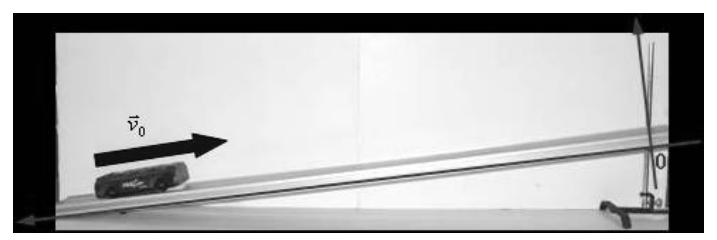

Fig. 4: Movimiento rectilíneo de un carrito con velocidad variable acercándose al origen del sistema de referencia (MRUA, análisis solamente del movimiento de subida).

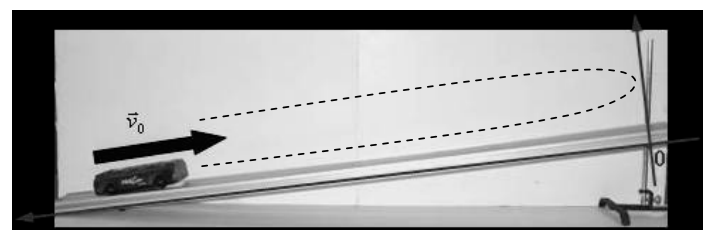

Fig. 5: Movimiento rectilíneo de un carrito con velocidad variable acercándose al origen del sistema de referencia y regresando al punto de partida (MRUA, análisis del movimiento de subida y de bajada). 


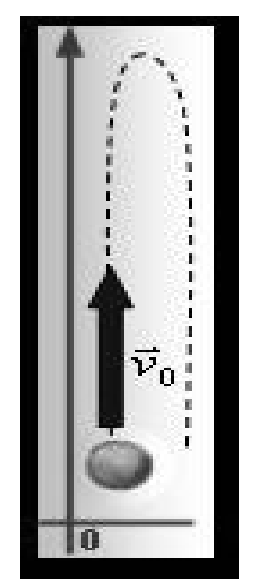

Fig. 6: Movimiento rectilíneo de una pelota con velocidad variable alejándose del origen del sistema de referencia y regresando al punto de partida (MRUA, análisis del movimiento de subida y de bajada del cuerpo).

Para el desarrollo de las clases de apoyo destinadas a los grupos experimentales se seleccionaron los siguientes recursos tecnológicos:

- DataStudio es un programa de recopilación, análisis y presentación de datos. Recopila y muestra los datos durante el experimento, mediante la conexión de los sensores a la interfaz y la configuración del software. Se pueden mostrar los datos en formato digital, analógico, gráfico o en modo osciloscopio. Las ventanas del programa se actualizan en tiempo real (mientras que se toman los datos). El software hace uso de interfaces y sensores para recopilar y analizar los datos, convirtiendo las prácticas en virtuales.

- VideoPoint es un software que permite recolectar datos de tiempo y posición a partir de video digital en la forma de "puntos de video", lo que se logra haciendo clic en la ubicación de los puntos de interés para cada cuadro en una película. Estos puntos pueden luego combinarse para llegar a otros cálculos, como la ubicación del centro de masa y distancia entre puntos. Es un software con el cual se pueden utilizar múltiples marcos de referencia para analizar movimiento relativo. Permite la integración de datos/gráficos, modelado de datos con ecuaciones, ajuste de datos. Permite realizar gráficos de velocidad, aceleración, momento y energía, en coordenadas polares y cartesianas, análisis de movimiento y se pueden exportar datos.

\section{RESULTADOS Y DISCUSIÓN}

Analizando los datos de las experiencias realizadas se observan logros estadísticamente significativos en los rendimientos de los alumnos que integran los grupos experimentales con respecto al grupo control. En ambos grupos experimentales se comprueba un impacto apreciable del uso de las TICs en la compresión de los gráficos de cinemática en los alumnos de Física I del 2006.

Consecuentemente, este trabajo ha permitido conocer el impacto que tiene el uso de las nuevas tecnologías en los procesos de enseñanza y aprendizaje en determinados tópicos de la física.

El cuestionario Test de Comprensión de Gráficos de Cinemática (TUG-K) (Beichner, 1994) fue aplicado al comienzo (pretest) y al final de la instrucción (postest). Con los datos obtenidos se calculó la ganancia normalizada (fracción de ganancia máxima posible), definida como (Hake, 1998):

$g=(<$ post $>-<$ pre $>) /(100-<$ pre $>)$

donde el signo < > indica el promedio de respuestas correctas de todo el curso para el pretest y postest, respectivamente. La ganancia normalizada permite comparar el grado de logro de la estrategia educativa en distintas poblaciones, independientemente del estado inicial de conocimiento. Es una medida intensiva de la ganancia obtenida y muy útil para comparar, por 
ejemplo, estudiantes secundarios con universitarios o de distintas instituciones. Los resultados obtenidos en las pruebas aplicadas a cada grupo se muestran en la Tabla 1.

Tabla 1: Resultados (porcentaje de repuestas correctas) del pretest, postest y ganancia para los grupos experimentales y control.

\begin{tabular}{|c|c|c|c|c|}
\hline $\begin{array}{c}\text { Curso Física 1 - 2006 } \\
(\mathrm{n}=62)\end{array}$ & $\begin{array}{c}<\text { pre> } \\
(1)\end{array}$ & $\begin{array}{c}<\text { post } \\
(2)\end{array}$ & $\begin{array}{c}<\text { ganancia } \\
(2)-(1)\end{array}$ & $<$ ganancia Hake $>$ \\
\hline $\begin{array}{c}\text { Grupo 1 - Control } \\
\text { Enseñanza tradicional } \\
(\mathrm{n}=21)\end{array}$ & $35,3 \%$ & $38,5 \%$ & $3,2 \%$ & $5,0 \%$ \\
\hline $\begin{array}{c}\text { Grupos 2- Experimental } \\
\text { Enseñanza con DataStudio } \\
(\mathrm{n}=20)\end{array}$ & $41,7 \%$ & $54,4 \%$ & $12,7 \%$ & $21,8 \%$ \\
\hline $\begin{array}{c}\text { Grupo 3 - Experimental } \\
\text { Enseñanza con Videopoint } \\
(\mathrm{n}=21)\end{array}$ & $33,5 \%$ & $45,4 \%$ & $11,8 \%$ & $17,8 \%$ \\
\hline
\end{tabular}

Mediante el análisis de los resultados obtenidos en este estudio, se pudo comprobar que se obtienen diferencias estadísticamente significativas en el rendimiento medio de los estudiantes un curso de Física de la FICES-UNSL, cuando en un grupo la estrategia de enseñaza se implementa utilizando un tratamiento demostrativo basado en recursos tecnológicos, como por ejemplo DataStudio o VideoPoint comparado con otro en el cual se realiza un abordaje tradicional.

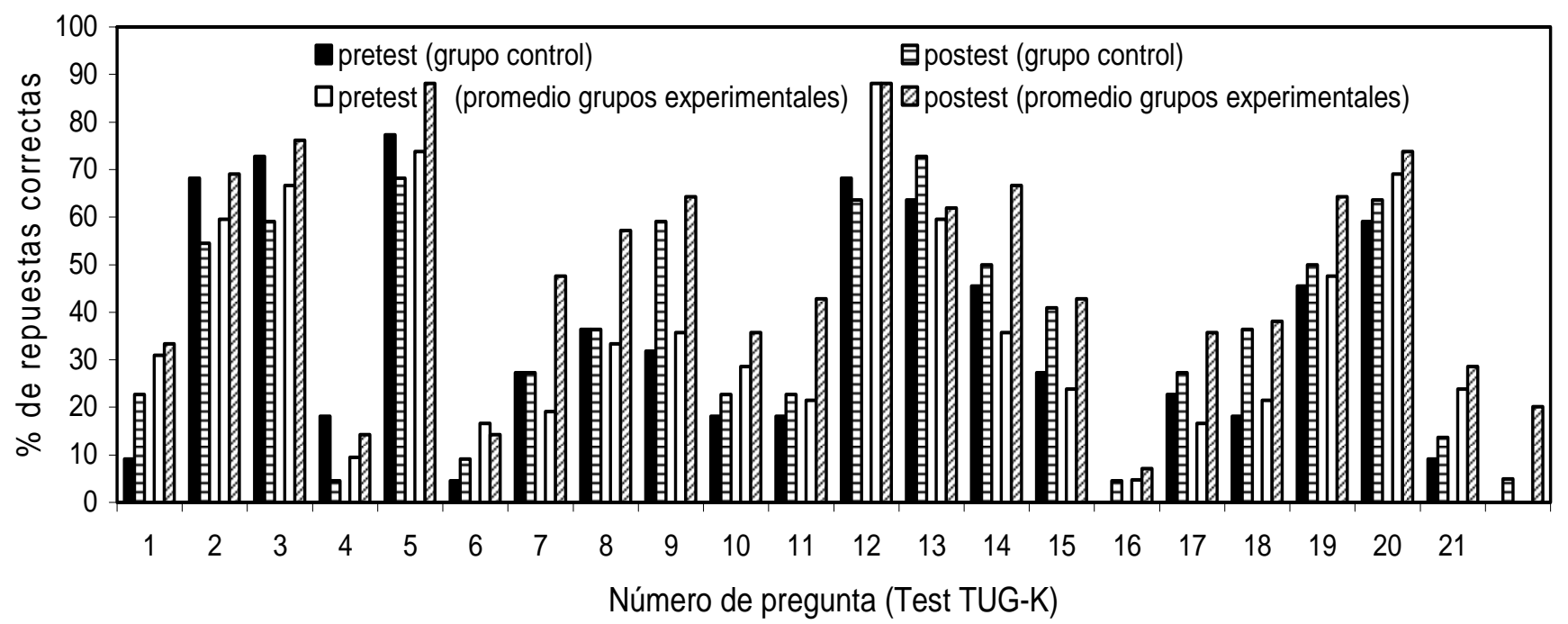

Fig. 7: Resultados comparativos sobre el rendimiento de los alumnos para cada una de las 21 preguntas del pretest y postest correspondiente al Test de Comprensión de Gráficos de

Cinemática (TUG-K) (Beichner), para el grupo control y para el promedio de los grupos experimentales. Las dos últimas barras indican la Ganancia Hake para cada población en estudio, grupo control y promedio grupos experimentales, respectivamente.

La ganancia relativa obtenida resulta un valor apreciable, si se analiza que en promedio un alumno que recibe una instrucción con recursos tecnológicos tiene un rendimiento del 20\% aproximadamente comparado con otro alumno que tuvo una instrucción utilizando una metodología tradicional (Fig. 7).

\section{CONCLUSIONES}

La experiencia en estudio se enmarca en los aspectos pedagógico-didácticos que fundamentan las prácticas docentes cuyas estrategias de enseñanza incluyen el uso de las TICs para propiciar aprendizajes comprensivos que contribuyan a la formación de los ingenieros. 
El uso de las tecnologías educativas son parte interdependiente de las prácticas pedagógicas desplegadas por los sujetos educativos, en tanto pueden ser incorporadas por el docente cuando pone en juego diferentes estrategias didácticas para promover el aprendizaje de un determinado contendido disciplinar en sus alumnos.

Se observa que la aplicación de los recursos tecnológicos para representar fenómenos físicos en entornos presenciales contribuye a mejorar el aprendizaje comprensivo de los conceptos de Física en estudiantes del primer año de las carreras de Ingeniería.

Finalmente, se adhiere a la idea de que al adoptar criterios de incorporación de las TICs, es necesario tener en cuenta que "las repercusiones de los nuevos desarrollos tecnológicos requieren ser estudiados desde una perspectiva pedagógica, ya que la transformación de las formas de enseñar no se produce por la renovación de los artefactos, sino por la reconstrucción de los encuadres pedagógicos de dicha renovación" (Maggio, 2000).

\section{REFERENCIAS}

Arons, A.; "A guide to Introductory Physics Teaching", Wiley, Nueva York, Estados Unidos (1990).

Beichner, R.; Testing student interpretation of kinematics graphs, American Journal of Physics: 62, 750-762 (1994).

Castorina, A.; "Los problemas conceptuales del constructivismo y sus relaciones con la ecuación", en Debates constructivistas por Baquero, R. et al., 21-45, Aique, Buenos Aires, Argentina (1998).

Coll, C.; "Aprendizaje escolar y construcción del conocimiento", Cap. 9, 189-205, Paidós, Barcelona, España (1990).

Hake, R.; Interactive-engagement vs traditional methods: A six-thousandstudent survey of mechanics test data for introductory physics courses, American Journal of Physics: 66, 64-74 (1998).

Maggio, M., "El tutor en la educación a distancia", en La educación a distancia por Litwin, E. (compiladora), Colección Agenda Educativa, Amorrortu Editores S.A, Buenos Aires, Argentina (2000).

McDermott, L., M. Rosenquist y E. van Zee; Student difficulties in connecting grapas and physics: Examples from kinematics, American Journal of Physics: 55, 6, 503-513 (1987).

Perkins, D.; "La persona-más: una visión distribuida del pensamiento y el aprendizaje", en Cogniciones distribuidas de consideraciones pedagógicas y educativas por Salomón, G. (compilador), Editorial Amorrortu, Buenos Aires, Argentina (1993).

Pontes, A.; Aplicaciones de las nuevas tecnologías de la información en la educación científica. $1^{a}$ Parte: Funciones y recursos. Revista Eureka sobre Enseñanza y Divulgación de las Ciencias: 2(1), 2-18 (2005).

Redish, E., J. Saul y R. Steinberg; Student expectations in introductory physics, American Journal of Physics: 66, 212-224 (1997).

Salomón, G.; "Cogniciones distribuidas", Editorial Amorrortu, Buenos Aires, Argentina (2001).

Vélez, G., "Aprender en la Universidad. La relación del estudiante universitario con el conocimiento", Universidad Nacional de Río Cuarto, Córdoba, Argentina (2002). 